\title{
Metal Injection Molding Process Parameters as A Function of Filling Performance of 3D Printed Polymer Mold
}

\author{
Junaid A. Qayyum ${ }^{1}$, Khurram Altaf ${ }^{1,}$, , A. Majdi A. Rani ${ }^{1}$, Faiz Ahmad ${ }^{1}$, Hafiz A. Qadir ${ }^{2}$ \\ and Waseem $\mathrm{Amin}^{3}$ \\ ${ }^{1}$ Center for Automotive Research and Electric Mobility, Department of Mechanical Engineering, \\ Universiti Teknologi PETRONAS, 32610 Seri Iskandar, Malaysia \\ ${ }^{2}$ Department of Chemical Engineering, NFC Institute of Engineering and Fertilizer Research, 38000 \\ Faisalabad, Pakistan \\ ${ }^{3}$ Interdisciplinary Centre for Advanced Material Simulations, Ruhr-Universität Bochum, 44801 \\ Bochum, Germany
}

\begin{abstract}
Metal injection molding (MIM) is a swift manufacturing process, which can produce complex and intricate parts with good repeatability and accuracy. However, to quickly address low-volume demands of customized MIM parts, manufacturing of mold could be a potential challenge. Typically, machined metal molds are used for MIM, but they are expensive and need more lead time. The machined metal mold becomes useless once the design is changed or requirement of MIM parts is met. Therefore, for MIM production of a low volume of highly customized parts, machined metal mold could be substituted by $3 \mathrm{D}$ printed polymer molds. However, knowledge of filling behavior of MIM feedstock in polymer mold is a grey area, which demands study to investigate the effects of injection parameters on mold filling. The present study investigates the effects of machine injection parameters on feedstock filling behavior in 3D printed polymer molds. An attempt has been made to determine the trend of feedstock filling in the polymer mold as a function of injection parameters. Further, the design of experiment (DOE) has been used to estimate the weight of injection parameters.
\end{abstract}

\section{Introduction}

Metal injection molding (MIM) is preferred for manufacturing of intricate components in a short time. Contrary to advance machining, in which a single intricate part could be manufactured after an intensive effort, MIM can produce more parts in less time. However, making of mold for MIM demands prolong lead-time and tiresome labour, therefore the justification of overall cost and lead-time could be hampered. For the potential applications involving customized designs like biomedical implants [1], automobile and aerospace spare parts etc. the manufacturing of mold could be a challenge. Nevertheless, 3D printed molds

\footnotetext{
*Corresponding author: khurram.altaf@utp.edu.my
} 
can fairly be used for low-volume production of customized parts [2]. Knowledge of mold filling up to certain confidence level is needed prior to proceeded downstream processes of prototyping and soft tooling. 3D printing could synergistically be integrated with polymer injection as metal injection molding (MIM) process to fabricate near-net shaped complicated and multifaceted parts [3]. Usually the surface of metal mold, if made through advance machining, is smooth. Nevertheless, molds made through fused deposition modelling (FDM) bear inherent downside of surface roughness. Therefore, right combination of injection parameters is needed to be determined for smooth running of the MIM cycle.

Hong et al. [4] studied mold filling behavior as function of time and then thermal contact resistance as function of position and time using short shot injection molding. Control of mold roughness and thermal resistance could contribute to superior filling. Lucchetta et al. [5] investigated filling of polymer feedstock in three types of coated inserts, viz. aluminum oxide, diamond like carbon and silicon oxide. Due to coating, reduction up to $8 \%$ was reported in flow resistance nevertheless, the resistance is also markedly dependent on the injection temperature.

Some researchers believe that increasing mold temperature could improve filling performance of 3D printed polymer mold [6]. Increased pressure and temperature of feedstock could introduce voids in the flow, thereby impairs the strength [7]. Zhang et al. [8] investigated effects of molding temperature and injection speed on the filling behavior of polyethylene based feedstock in mold insert, made from methyl methacrylate and acryl amide containing photopolymer. Two levels of three parameters viz. mold temperature, injection temperature and injection speed were varied and the results were analyzed using full factorial design of experiment (DOE). It was learnt that at lower mold temperature and injection speed, dimensional consistency is more pronounced. Kim et al. [9] comparison of filling behavior of polycarbonate/LexanTM and SS 316L feedstock in multi-cavity mold system using shortshot IM. Injection time was varied to $4 \mathrm{sec}$ in increments of $0.5 \mathrm{sec}$ and filling behavior of multi-cavity mold was studied. The study however could be extended to manifest filling behavior in polymer molds. Barrier et al. [3] numerically modeled filling of multi-cavity mold as function of mold design and validated using finite elemental approach. Threshold injection pressure to completely fill the mold cavity was thus evaluated. The study however could be extended to include optimization of injection temperature for complete filling of mold cavity.

Junaid et al. [2, 10] successfully carried out MIM of copper based feedstock through FDM made polymer molds. However, the study was highly focused on performance of polymer mold to successfully undergo MIM. To develop overall manufacturing process sustainable, a comprehensive knowledge of effects of molding parameters on the mold filling behavior is inevitable. The present study is an attempt to investigate mold-filling behavior as a function of injection parameters. Some authors $[4,5]$ studied filling behavior of polymer feedstock in modified metal molds, however the converse, meaning that filling behavior of metal based feedstock in polymer molds still remains a grey area. The present study focuses on filling behaviour of copper-based feedstock in FDM made polymer mold as function of injection pressure and temperature.

\section{Methodology}

In the present study, copper-based feedstock was used with polyethylene as binder, steric acid and wax as surfactant. The copper powder and binder were mixed in ratio of $59 \%$ and $41 \%$ respectively. Critical solid powder loading was made according to ASTM D-281-31 and calculated through the following expression as $69 \%$ [11]. $V_{f}$ and $V_{o}$ are volumes of powder and oleic acid respectively, the powder loading could be given as [12]; 


$$
\text { Powder loading } \%=\frac{V_{f}}{V_{f}+V_{0}} \times 100
$$

The mold assembly contained a mold insert, housed in mold block, 3D model of which is shown in figure 1. Mold block and mold insert were manufactured using fused deposition modeling (FDM) as fully dense solid part to sustain a significant number of MIM cycles. The design of the mold was according to standard MIM part, made from proprietary acrylonitrile butadiene styrene (ABS M-90) material.

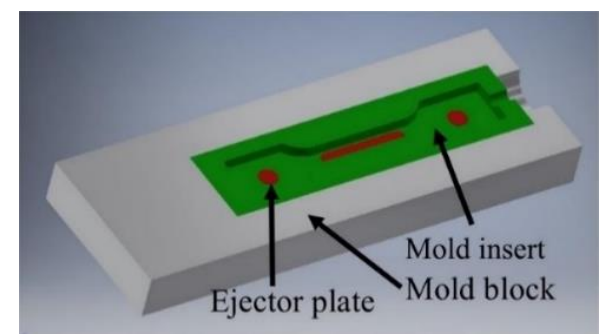

Fig. 1. 3D model of mold assembly.

\subsection{Design of experiment}

Response surface methodology (RSM) was used as design of experiment (DOE) to investigate the filling behavior of copper-based feedstock in 3D printed polymer mold, as function of injection pressure and temperature. Three levels of these two parameters were investigated in the study. Table 2 provides the list of parameters and magnitudes of their varying levels. Table 3 provides the combination of different levels of parameters as defined by RSM. Mass of feedstock filled was investigated as the function of injection pressure and injection temperature of MIM machine. For each combination three MIM cycles were run and average value was selected for mass filling analysis.

Table 1. Factors influencing mold filling

\begin{tabular}{|l|c|c|c|}
\hline \multirow{2}{*}{ Factors } & \multicolumn{3}{|c|}{ Levels } \\
\cline { 2 - 4 } & $\mathbf{- 1}$ & $\mathbf{0}$ & $\mathbf{1}$ \\
\hline Injection temperature (C) & 150 & 175 & 200 \\
\hline Injection pressure (bar) & 2 & 3.5 & 5.0 \\
\hline
\end{tabular}

\subsection{Injection molding cycles}

Injection molding was carried out using KSA100 vertical injection molding machine. The machine has maximum injection pressure and temperature of $10 \mathrm{bar}$ and $220^{\circ} \mathrm{C}$, however due to constraints of the feedstock under study, MIM cycles were conducted at maximum of 5 bar and $200{ }^{\circ} \mathrm{C}$ respectively. The maximum loading volume of mandrel is $6.6 \mathrm{~kg}$ and screw diameter is 5 in. MIM machine was operated on manual mode and injection was carried out for about $10 \mathrm{sec}$ to fill the cavity at maximum and to avoid any possibility of short-shot.

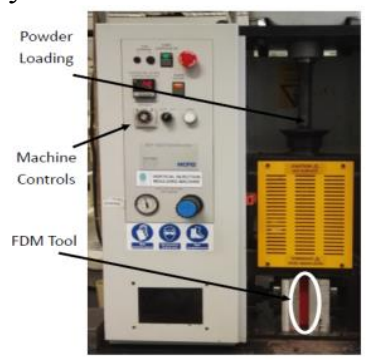

Fig. 2. Metal injection molding machine. 


\subsection{Analysis of MIM feedstock filling}

Prior to MIM cycle, the mold insert was weighed on an electric balance having resolution of $1 \mathrm{mg}$. After every MIM cycle, once the feedstock has been completely solidified, the mold insert was ejected from the mold block and weighed again. The mold filling was measured as mass difference of as filled and empty mold insert. Hence, mold filling was figured as function of temperature and pressure of injection molding machine. Estimated dependence of MIM parameters on filling of polymer molds was qualified using regression analysis.

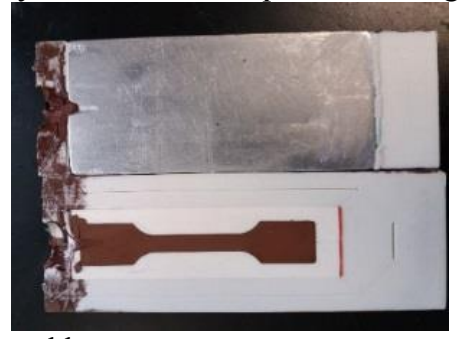

Fig. 3. MIM using 3D printed mold assembly.

\section{Results and discussion}

\subsection{Regression analysis}

Table 4. Response surface methodology results for mold filling

\begin{tabular}{|c|c|c|c|c|c|c|c|c|}
\hline $\begin{array}{c}\text { Sr. } \\
\text { No. }\end{array}$ & StdOrder & RunOrder & PtType & Blocks & $\mathbf{T}$ & $\mathbf{T}$ & $\mathbf{P}$ & Mass \\
\hline $\mathbf{1}$ & & & & & ${ }^{\circ} \mathrm{C}$ & $\mathrm{K}$ & bar & gm \\
\hline $\mathbf{2}$ & 10 & 2 & -1 & 1 & 175 & 448 & 2 & 16.9 \\
\hline $\mathbf{3}$ & 4 & 3 & 1 & 1 & 200 & 473 & 5 & 19.901 \\
\hline $\mathbf{4}$ & 8 & 4 & -1 & 1 & 175 & 448 & 5 & 19.586 \\
\hline $\mathbf{5}$ & 6 & 5 & -1 & 1 & 200 & 473 & 3.5 & 17.186 \\
\hline $\mathbf{6}$ & 12 & 6 & 0 & 1 & 175 & 448 & 3.5 & 16.96 \\
\hline $\mathbf{7}$ & 1 & 7 & 1 & 1 & 150 & 423 & 2 & 14.526 \\
\hline $\mathbf{8}$ & 13 & 8 & 0 & 1 & 175 & 448 & 3.5 & 16.986 \\
\hline $\mathbf{9}$ & 11 & 9 & 0 & 1 & 175 & 448 & 3.5 & 17.086 \\
\hline $\mathbf{1 0}$ & 5 & 10 & -1 & 1 & 150 & 423 & 3.5 & 14.886 \\
\hline $\mathbf{1 1}$ & 2 & 11 & 1 & 1 & 200 & 473 & 2 & 16.926 \\
\hline $\mathbf{1 2}$ & 9 & 12 & 0 & 1 & 175 & 448 & 3.5 & 17.067 \\
\hline $\mathbf{1 3}$ & 3 & 13 & 1 & 1 & 150 & 423 & 5 & 16.8 \\
\hline
\end{tabular}

\subsection{Effect of injection temperature on mold filling}

When injection pressure was kept constant at 2 bars, filled mass was observed as $14.526 \mathrm{~g}$, $16.9 \mathrm{~g}$ and $16.926 \mathrm{~g}$, corresponding to injection temperatures of $423 \mathrm{~K}, 448 \mathrm{~K}$ and $473 \mathrm{~K}$ respectively. Keeping pressure at 3.5 bars, filled masses were calculated as $14.886 \mathrm{~g}, 17.0146$ $\mathrm{g}$ and $17.186 \mathrm{~g}$ against the above mentioned magnitudes of injection temperatures respectively. Finally, for 5 bar injection pressure, filled masses was calculated as $16.8 \mathrm{~g}$, $19.586 \mathrm{~g}$ and $19.901 \mathrm{~g}$ for the above mentioned temperatures respectively. At all injection pressure values, increase in temperature initially contributed to increase in mold filling however, too much increase did not significantly augment mold filling. It could have been assumed that injection temperature should be kept higher to let the heat be properly distributed in the mold cavity to allow smooth subsequent injection molding cycles 
nevertheless, the experimental findings reveal that such increase could be much significant. This is attributed to the fact that heat is not dissipated quickly due to superior heat insulation properties of the polymer mold. Consequently, feedstock overheating, kept as a margin to compensate heat loss during injection molding, becomes unnecessary for polymer molds.

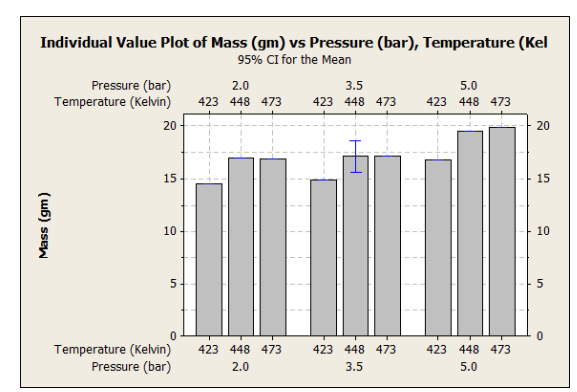

(a)

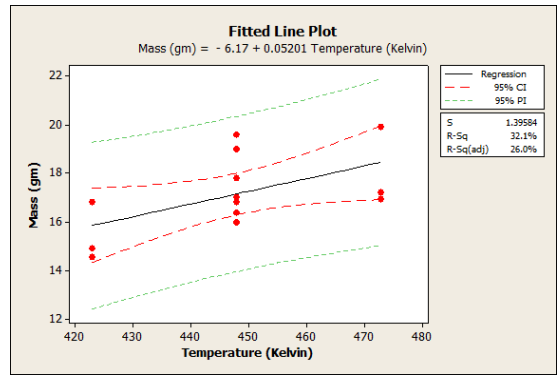

(b)

Fig. 4. (a) Individual plot and (b) fitted plot explaining mass filled as function of injection temperature

\subsection{Effect on injection pressure on mold filling}

When injection temperature was fixed at $423 \mathrm{~K}$, mold filling of $14.526 \mathrm{~g}, 14.886 \mathrm{~g}$ and 16.8 $\mathrm{g}$ were observed corresponding to injection pressure values of 2 bars, 3.5 bars and 5 bars respectively. At injection temperature of $448 \mathrm{~K}$ and $473 \mathrm{~K}$, mold fillling of $16.9 \mathrm{~g}$, 17.0146 $\mathrm{g}$ and $19.586 \mathrm{~g}$ were observed for former whereas $19.926 \mathrm{~g}, 17.186 \mathrm{~g}$ and $19.901 \mathrm{~g}$ for the later, corresponding to above values of injection pressures. Contrary to effect of injection temperature, where the increase was more prominent initially and less noticeable after further increase, the effect of injection pressure was observed less significant initially and more significant upon subsequent increase.

It could be concluded that higher injection pressure and moderately higher injection temperature are feasible for injection molding in polymer molds. Increasing the injection pressure increases the mold filling. The increasing effect of mass filling is very small for lower magnitudes of pressure, however at higher values of pressure, mass filling is increased significantly.

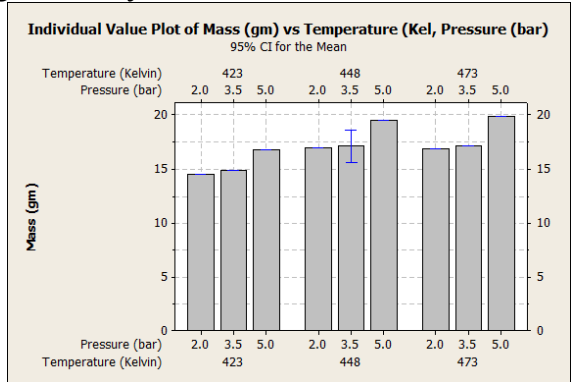

(a)

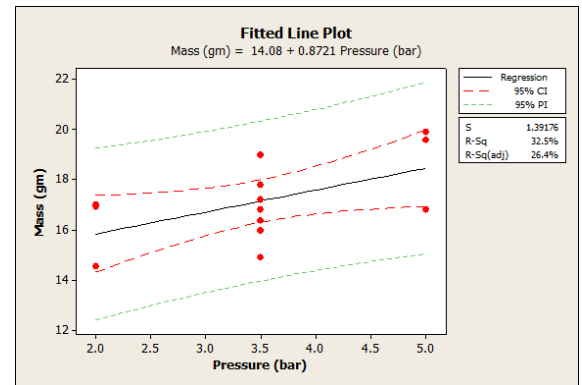

(b)

Fig. 5. (a) Individual plot and (b) fitted plot explaining mass filled as function of injection pressure

The over all contour plot of mass filing is also in accordance with the individual effects of injection temperature and pressure on the mass filling behavior (fig. 6). 


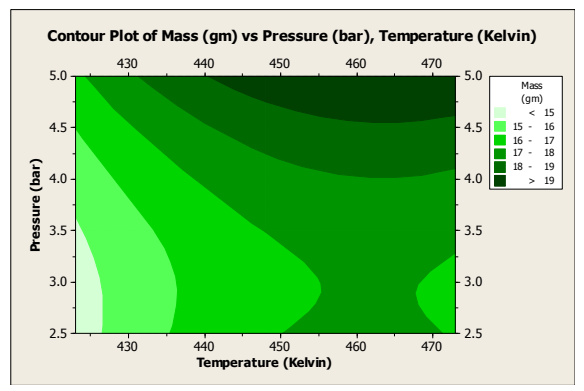

Fig. 6. Contour plot of Mass Vs Injection pressure and temperature

\section{Conclusion}

It could be observed that injection temperature and injection pressure effect the filling behavior of the mold cavity. Among the factors governing mold filling, temperature and pressure of feedstock at the time of injection are very important. Knowledge of effects of injection temperature and pressure on mold filling could be helpful in optimal design of the overall MIM cycle. Up to certain magnitudes of temperature and pressure, the magnitude of effect on mold filling changes, however the overall trend remains the same. Following conclusions could be drawn from the above study;

- Injection pressure and injection temperature are principal factors which govern the filling of feedstock in the mold cavity

- Injection pressure is however more significant parameter than injection temperature, when filling of polymer mold is concerned

The authors acknowledge financial support from Universiti Teknologi PETRONAS (UTP) Malaysia to conduct the research.

\section{References}

1. L. Melgoza, Rapid Prototyping Journal. 20(1): p. 2-12. (2014).

2. J. A. Qayyum, ARPN Journal of Engineering and Applied Sciences. 12(22): p. 64306434 (2017).

3. T. Barrière, Journal of materials processing technology. 125: p. 518-524 (2002).

4. S. Hong, International Journal of Heat and Mass Transfer, 87: p. 222-236 (2002).

5. G. Lucchetta, CIRP Annals-Manufacturing Technology, 65(1): p. 537-540 (2016).

6. M. Kurt, Materials \& Design, 30(8): p. 3217-3224 (2009).

7. M. Sardarian, Ceramics International, 43(6): p. 4785-4793 (2017).

8. Y. Zhang, Journal of Manufacturing Processes, 27: p. 138-144 (2017).

9. J. Kim, Powder Technology, 257: p. 124-131 (2014).

10. K. Altaf, METALS, 8 (6) (2018)

11. S. V. Atre, S.V., SAE Technical Paper (1998).

12. M. R. Harun, Applied Mechanics and Materials. Trans Tech Publ. (2011). 